\title{
Desiguales, pobres y excluidas. Lecciones metodológicas desde la (ausente) perspectiva de género
}

\section{Miguel Ángel Mateo Pérez}

Universidad de Alicante. Departamento de Sociología II

Ap. 99. 03080 Alicante

Data de recepció: febrer 2001

Data d'acceptació: març 2001

\section{Resumen}

El presente trabajo es una reflexión conceptual y metodológica sobre la pobreza y la perspectiva de género, sus ausencias en las investigaciones más recientes mundiales y españolas. Se estudian y reformulan los conceptos de desigualdad, pobreza y exclusión, así como sus implicaciones metodológicas traducidas a indicadores. Se concluye con una propuesta metodológica para la inclusión de la perspectiva de género en el estudio de la pobreza.

Palabras clave: pobreza, desigualdad, exclusión social, perspectiva de género, metodología.

\section{Abstract. The unequally treated, impoverished and excluded women}

The present work is a conceptual and methodological reflection on the poverty and the perspective of gender, their absences in the most recent global research. We study the concepts of inequality, poverty and exclusion, as well as their methodological implications translated to indicators. We conclude with a methodological approach for the inclusion of the perspective of gender in the study of the poverty.

Key words: poverty, inequality, social exclusion, perspective of gender, methodology.

\section{Sumario}

\section{Introducción 4. Perspectivas de género y procesos}

2. Disciplinas. Enfoques y desenfoques

3. Definiciones. ¿Desiguales, pobres

y/o excluidas? de empobrecimiento

5. Conclusiones y alternativas para una presencia nada clara

* Este trabajo ha sido posible gracias a la financiación del Ministerio de Trabajo y Asuntos Sociales a través del Instituto de la Mujer, al proyecto dirigido por José María Tortosa Indicadores dinámicos para el estudio del empobrecimiento de las mujeres (REF: MI-0003). 
Para mí, la pobreza real es una mujer pobre.

Baboucar Gaye ${ }^{1}$

\section{Introducción}

Desde hace ya algún tiempo se está trabajando sobre la pobreza, tanto como reflexiones sobre los menos afortunados han producido (y reproducido) las sociedades que tomemos como referencia (atención a los caprichos del lenguaje, sobre todo a los géneros más empleados).

Que siempre hemos tenido pobres ${ }^{2}$ es una cuestión más que evidente. Pero no siempre los hemos definido de igual manera, no siempre hemos considerado los mismos elementos o características comunes que han de tener los pobres para serlo. Hoy, alguien que no esté evangelizado, no tiene el título de pobre homologado por estudios de carácter internacional, por ejemplo (ni siquiera forzando los términos con aquello de "pobres de espíritu»). Pero al margen de estas consideraciones, que son más importantes de lo que habitualmente pensamos, desde las ciencias sociales se ha sistematizado el estudio de aquéllos que no disfrutan de las mismas condiciones de vida que la distribución normal de la población, en una sociedad de referencia concreta, en un tiempo concreto, a partir de los procesos de industrialización (siglos XVIII y XIX, fundamentalmente) en Europa, para ser más precisos $^{3}$.

Las transformaciones sociales de la industrialización centraron el análisis de muchos científicos sociales en esa no tan nueva realidad social que era la pobreza severa, miseria de los trabajadores ${ }^{4}$ y los problemas que se derivaban de aquellas situaciones extremas de empobrecimiento, así como en los procesos de acumulación y su lógica. Esto hace que los estudios sobre la pobreza adquieran un compañero inseparable, el estudio de la riqueza, que incluso perdurará hasta nuestros días en estos términos ${ }^{5}$, y en otros, como desarrollo, modernización, crecimiento...

1. "61 ways of looking at poverty", en Choices, The Human Development Magazine, 1996.

2. Por citar algún trabajo reciente en esa dirección, ver TorTosa, J.M. (1999). Pobreza, desarrollo y prospectiva. PYDLOS, Universidad de Cuenca (Ecuador) y MUÑOZ DE BUSTILLO, R.; EsTEVE, F. (1998). «Marco general: pobreza y economía de mercado». En VV.AA. (1998). Las condiciones de la población pobre. Madrid: FOESSA.

3. Miles, I.; IRvine, J. (1982). The poverty of progress. Changing ways of life in industrial societies. Pergamon Press.

4. Engels, F. (1976). La situación de la clase obrera en Inglaterra. Madrid: Akal.

5. Ver el trabajo de Cáritas en esta dirección y, en particular, la publicación del número 76 de la revista Documentación Social que tiene precisamente este título "Riqueza y pobreza», julio-septiembre de 1989. Ver también GILDER, G. (1989). Riqueza y pobreza. Madrid: Instituto de Estudios Económicos. 
Los nuevos pobres ${ }^{6}$ las nuevas pobres ${ }^{7}$ o pobrezas, tienen de nuevos algunas matizaciones que, en algunos casos, sí definen situaciones totalmente nuevas, pero que en otros tienen más continuación que ruptura, gracias a uno de los rasgos de los procesos de empobrecimiento: la permanencia.

De todas formas, no creo pertinente la realización de un análisis general sobre todos los estudios y aproximaciones que afectan a la pobreza y la convierten en objeto de investigación y reflexión teórica o empírica. Sin embargo, para comprender algunas de las cosas que en estas páginas se irán mencionando, es necesario detenerse en algunas visiones más o menos generales sobre la pobreza, al menos en este siglo XX que ya llega a su fin, sin un consenso sobre qué es y cómo afrontar el empobrecimiento masivo de las personas, independientemente del sexo de las mismas. Más tarde nos detendremos en los conceptos de desigualdad, pobreza y exclusión social, para finalizar con las diferentes aportaciones que la perspectiva de género realiza o puede potencialmente realizar, en el estudio del empobrecimiento.

\section{Disciplinas. Enfoques y desenfoques}

Los objetivos de los que se parte en la investigación sobre pobreza consolidan una u otra forma de entender la realidad social en general. En el caso de los estudios de pobreza realizados desde la perspectiva de la economía, generalmente se ha pretendido medir la pobreza y, como mucho, utilizar los datos cuantitativos para aplicar análisis descriptivos y en muy pocos casos explicativos ${ }^{8}$. Las unidades empleadas desde esta perspectiva son siempre agregados (estados o niveles un poco inferiores como los hogares) y variables muy discutidas como el PIB o PNB o simplemente las rentas familiares?. En el otro extremo tenemos disciplinas como el trabajo social, cuyas unidades son las más concretas posibles (personas pobres) y en cuyas investigaciones (más prácticas que teóricas) buscan explicar las situaciones problema para actuar a ese nivel de concreción ${ }^{10}$ (tabla 1).

El estudio de la pobreza desde la perspectiva de una sola disciplina es un problema epistemológico de partida muy importante. La supuesta síntesis más pertinente en el estudio de la pobreza podría venir desde una disciplina integradora como es la sociología. Sin embargo, nuestra disciplina no ha ofrecido hasta el momento una definición de pobreza que satisfaga todas las perspectivas.

6. Oltra, B. «Prólogo». En Casado, D. (1990). Sobre la pobreza en España: 1965-1994. Barcelona: Hacer.

7. Pressman, S. (1998). "The gender poverty gap in developed countries: causes and cures». Social Science Journal, 35, 2, abril, p. 275-286.

8. Ver los trabajos para España de Jesús Ruiz Huerta y Rosa Martínez, (1994). «La pobreza en España: ¿Qué nos muestran las EPF?». Documentación Social, 96, p. 15-110.

9. Para más referencias sobre ese en particular, ver SEN, A. (1995). Nuevo examen de la desigualdad. Madrid: Alianza.

10. EsCARTín, M.J.; SuÁreZ, E. (1994). Introducción al trabajo social. Alicante: Anagrama. 
Tabla 1. Disciplinas que estudian la pobreza.

\begin{tabular}{|c|c|c|c|}
\hline Disciplinas & Economía & Trabajo social & Sociología \\
\hline Perspectiva & Abstracta & Concreta & Abstracta \\
\hline Objeto & Agregados & Personas & Agregados/personas \\
\hline Metodología & Cuantitativa & Cualitativa & $\begin{array}{l}\text { Cuantitativa } \\
\text { (predominante) } \\
\text { Cualitativa }\end{array}$ \\
\hline Causas & No definidas (el pobre) & No definidas (sistema) & Sistema de causas \\
\hline Políticas & Reducir agregados & Mejorar individuos & Reducir agregados \\
\hline
\end{tabular}

Es posible que la definición sociológica de la pobreza sea más difícil que la aproximación exclusivamente economicista. Pero es precisamente fruto de esa dificultad de donde surgen las preguntas. Y son esas preguntas las que nos hacen pensar en posibles enfoques alternativos a los que en la actualidad disponemos. Al menos, lo que sí sabemos es que las investigaciones más rigurosas y completas sobre pobreza pasan por una necesaria interdisciplinariedad.

Podemos sistematizar algunos de los estudios sobre pobreza desde 1940 hasta hoy en dos ejes. Uno que muestre los niveles de agregación de las variables y unidades (desde el máximo: estados; hasta el mínimo: personas; pasando por grupos y hogares) y otro que muestre una serie de dimensiones de la pobreza (sobre las que más tarde volveré) que vaya desde la pobreza monetaria (dimensión económica ligada a las rentas o, dicho de otra manera, a la privación clásica), la dimensión política (que tiene que ver con la participación), la dimensión cultural (que podemos resumir en la capacitación) hasta la dimensión más puramente social (que se puede denotar en el extremo opuesto de la pobreza monetaria y que sin duda se acerca más al concepto de exclusión social y a la (des)igualdad de oportunidades).

Los estudios sobre pobreza en España en la década de los ochenta presentan algunos rasgos comunes ${ }^{11}$, aunque resultados diferentes. Esto puede achacarse a la aún escasa disponibilidad de datos cuantitativos ${ }^{12}$, por un lado, y, por otro, a problemas conceptuales. Esta última indicación es un poco más discutible, aunque para España no existe una línea oficial de pobreza (sí para EEUU, por ejemplo ${ }^{13}$ ) y la asimilación de las normativas de la UE ya se produce a mediados de la década de los noventa adecuando la definición operativa de pobreza a los estándares europeos.

11. LLES, C. (1989). «Los estudios sobre pobreza y exclusión social en la España de los 80». Documentación Social, 76, julio-septiembre, p. 173-188.

12. VILA, L. (1991). «Ética y economía: el ejemplo de la pobreza». ICE, 691, marzo.

13. BradshaW, J.; BouWKNEgT, L.; Holmes, H. (1996). «In Search of a Representative Measure of poverty». En SMITH, E. (ed.) (1996). Measuring outcome in the public sector. Taylor\&Francis, capítulo 9. 
Estudios que, desde los puramente económicos basados en la Encuesta de Presupuestos Familiares ${ }^{14}$, pasando por el V Informe Foessa ${ }^{15}$, hasta llegar a encuestas específicas a grupos empobrecidos ${ }^{16}$, éstas ya muy recientes, han dejado una estela de investigaciones paralelas que abren camino para otras investigaciones empíricas (y sobre el concepto).

Así pues, diferentes disciplinas y diferentes estudios sobre pobreza, su diversidad y complejidad, así como las diferencias entre unos y otros (y eso que tienen como objeto (en principio) una misma materia, la pobreza) podría ser una primera reflexión interesante.

$\mathrm{Si}$, en definitiva, investigamos sobre pobreza y no nos ponemos de acuerdo en qué unidades tomamos, ni en qué variables, ni en qué dimensiones de la realidad social incidimos (si es que acaso reconocemos que la realidad social es compleja y no todo se reduce al dígito economicista), suponemos que estamos ante un problema conceptual de primera magnitud.

La respuesta ante estos problemas no consiste únicamente en abogar por una solución ecléctica (sumemos todas las aportaciones que sobre una misma materia se realizan y la resultante será el resumen complejo y completo de dicha materia). En este caso, cuando hablamos de pobreza, creemos necesaria una reflexión conceptual desde la base, esto es la empiria, para evitar soluciones eclécticas, cómodas por otra parte, y empezar a trabajar con metodologías e indicadores si no nuevos, sí basados en unos nuevos conceptos o en nuevas aportaciones, como por ejemplo las derivadas de la perspectiva de género. Empecemos por las definiciones.

\section{Definiciones. ¿Desiguales, pobres y/o excluidas?}

Cuando estudiamos la desigualdad, no solemos discutir sobre el concepto, particularmente cuando estudiamos la desigualdad de rentas. Sin embargo, más problemas tenemos cuando nos referimos a conceptos como igualdad (o desigualdad) de oportunidades ${ }^{17}$ o si utilizamos el término desigualdad con matices más sociológicos.

Está claro que dos hogares son diferentes con respecto a sus ingresos monetarios cuando la distribución de éstos en el hogar 1 es diferente que en la del hogar 2. Pero cuando tratamos de ir un poco más allá de lo meramente descriptivo y buscamos explicaciones sociológicas a dicha distribución diferente, encontramos más que problemas para poder mostrar un consenso de variables sociológicas de desigualdad ${ }^{18}$. Pero no todo es tan claro. Por ejemplo, si que-

14. Ruiz-Castillo, J. (1987). La medición de la pobreza y la desigualdad en España, 1980-1981. Madrid: Banco de España.

15. VV.AA. (1995). V Informe Sociológico sobre la Situación Social en España. Madrid: FOESSA.

16. VV.AA. (1998). Las condiciones de vida de la población pobre. Madrid: FOESSA.

17. REES, T. (1998). «Social exclusion and equal opportunities». International Planning Studies, 3, 1, p. 15-20.

18. Para un análisis general de variables de desigualdad social desde diferentes niveles de análisis, ver Alaminos, A.; BAS, E.; MateO, M.A. (1997). Nuevas tecnologías, nuevas sociedades. Alicante: Instituto de Cultura Juan Gil-Albert. Para un modelo completo y amplio 
Tabla 2. Cuatro visiones (y cuatro conceptos) sobre la pobreza mundial (1940-1990).

\begin{tabular}{|c|c|c|c|c|}
\hline & Visión 1 & Visión 2 & Visión 3 & Visión 4 \\
\hline Definición & Renta & Niveles de subsistencia & «El otro desarrollo» & Desarrollo humano \\
\hline Tiempo & $1940-1950$ & Década de los sesenta & Década de los setenta & Década de los noventa \\
\hline Objeto & Mundial & Mundial & Mundial/local/sistema & Mundial/sistema \\
\hline Materiales & $\begin{array}{l}\text { Rentas } \\
\text { Consumo }\end{array}$ & $\begin{array}{l}\text { Niveles de subsistencia } \\
\text { absolutos y universales }\end{array}$ & $\begin{array}{l}\text { Necesidades humanas } \\
\text { básicas, sociales }\end{array}$ & $\begin{array}{l}\text { Necesidades } \\
\text { humanas }\end{array}$ \\
\hline Principios & $\begin{array}{l}\text { Incremento de } \\
\text { rentas para } \\
\text { consumir }\end{array}$ & $\begin{array}{l}\text { Alcanzar niveles de vida } \\
\text { como los países } \\
\text { desarrollados }\end{array}$ & $\begin{array}{l}\text { Satisfacción de las } \\
\text { necesidades humanas }\end{array}$ & $\begin{array}{l}\text { Satisfacción } \\
\text { necesidades }\end{array}$ \\
\hline Políticas & $\begin{array}{l}\text { Locales } \\
\text { (Estado) }\end{array}$ & Locales (Estado) & $\begin{array}{l}\text { Locales (Estado) } \\
\text { Cambios estructurales } \\
\text { a nivel mundial }\end{array}$ & $\begin{array}{l}\text { Locales (estados) } \\
\text { Globales } \\
\text { (¿multinacionales?) } \\
\text { Globales (¿ONG?) }\end{array}$ \\
\hline
\end{tabular}

remos analizar la desigualdad dentro de los hogares, atendiendo a la distribución (o mejor, redistribución) de las rentas por género, comprenderemos las dificultades que ofrecen los datos para poder realizar dicho trabajo.

Sobre el concepto de pobreza tenemos más elementos para poder señalar la falta de consenso, no sólo en su definición operativa y empírica, sino también en las variables que forman y explican las situaciones de empobrecimiento. Aunque no es momento para realizar un análisis detallado sobre las diferentes visiones conceptuales y variaciones sobre la pobreza como concepto sociológico $^{19}$, sí conviene señalar cuatro visiones sobre la pobreza entendida como una realidad mundial que abarca un periodo de tiempo desde 1940 hasta nuestros días y hacer referencia a lo que creemos podría ser un corpus teórico consensuado para la investigación sobre pobreza (tabla 2).

Hay que señalar que, además de estas visiones o paradigmas conceptuales sobre la pobreza como realidad social mundial, el debate ha seguido (y sigue) también por otros caminos: definición multidimensional o economicista de la pobre$\mathrm{za}^{20}$, dimensión absoluta o relativa u objetiva o relativa ${ }^{21} \mathrm{y}$, sobre todo, en la per-

sobre las desigualdades sociales en un contexto sociológico, ver Oltra, B. (1996). Modelos. Alicante: Editorial Club Universitario. Desde una visión de estructura de clases, ver los trabajos publicados bajo la coordinación de Rodolfo Gutiérrez en el número 75 de la Revista Española de Investigaciones Sociológicas, julio-septiembre, 1996.

19. Para ello, ver los estudios siguientes: RuSPINI, E. (1998). «From Rowntree to Panel Survey: A transition that has transformed the concept of poverty». Sociologia e Ricerca Sociale, 19, 55, p. 93-123; HARVEY, D.; REED, M. (1996). «The culture of poverty: an ideological analysis». Sociological perspectives, 39, 4, invierno, p. 465-495; VeIT-WILSON, J. (1986). «Paradigms of poverty: A rehabilitation of B.S. Rowntree». Journal of Social Policy, 15, 1, enero, p. 69-99; y, por último y tomando el caso de América Latina, VenANZI, A. (1998). The concept of poverty in Sociological Research: the case of latin america. ISA.

20. PNUD (1999). Human Development Report 1999. Nueva York: Oxford University Press.

21. BM (1999). World Development Report 1999/2000. Nueva York: Oxford University Press. 
tinencia de los diferentes indicadores empíricos para su medición ${ }^{22}$. Sin embargo, ser pobre, vivir en la pobreza, sigue siendo uno de los estigmas más importantes para quien lo padece ${ }^{23}$. Contrario a bienestar, riqueza o desarrollo humano, la pobreza, el empobrecimiento, en definitiva, la imposibilidad real de ser y compartir los beneficios que conlleva vivir en una sociedad humana organizada, es una situación en la que ninguno de nosotros quisiéramos estar. A nuestro entender, la cuestión de la pobreza no debe ser reducida a unas cuantas dimensiones.

Podemos definir la pobreza como un proceso ${ }^{24}$ (por lo tanto, con carácter dinámico) en el que las necesidades humanas consideradas básicas ${ }^{25}$ (salud y autonomía) no pueden satisfacerse de forma prolongada en el tiempo e involuntariamente ${ }^{26}$. Entonces, si entendemos la pobreza (proceso de empobrecimiento desde ahora) en los términos anteriormente enunciados, la verdad es que el debate entre conceptos supuestamente antagónicos como pobreza y exclusión social se hace inútil. Aunque en principio hay diferencias entre ambos conceptos (diferencias de origen y de matices ${ }^{27}$, diferencias conceptuales ${ }^{28}$ que tratan de separar un término de otro), el éxito de este último término en la literatura actual (tanto oficial como científica, que en muchos casos tienden a coincidir) es, desde nuestro punto de vista, un intento de suavizar la terminología en la caracterización de los problemas sociales. Así, prácticamente se podrían considerar como sinónimos, al menos en esencia conceptual, aunque, como bien señalan trabajos anteriores ${ }^{29}$, la pobreza ha sido considerada como

22. Mateo, M.A.; Penalva, C. (2000). «Per al mesurament de la desigualtat, pobresa i exclusió. Limitacions, propostes tècniques i alternatives». Revista Catalana de Sociologia, 11; RuIZCastillo, J.; Del Río, C. (1997). TIPs for poverty analysis. The case of Spain, 1980-81 to 1990-91. Universidad Carlos III de Madrid (Working Papers).

23. Tezanos, J.F. (1999). Tendencias de exclusión social en las sociedades tecnológicas. El caso español. Madrid: Sistema.

24. Choudhury, S.; LeONeSIO, M. (1997). «Life-cycle aspects of poverty among older women». Social Security Bulletin, 60, 2, p. 17-36.

25. Aguiar, F. (1996). «Teorías de las necesidades: una tipología». Revista Internacional de Sociologia, 13, p. 135-146; BASU, M. (1996). "Humanization of development: the question of Basic Needs». Social Action, 46, 3, p. 249-260; BouZADA, X. (1994). «Sobre las necesidades sociales y culturales: entre la necesidad constatada y la necesidad construida». Papers, 44, p. 53-76.

26. Doyal L.; Gough, I. (1996). Teoría de las necesidades humanas básicas. Barcelona: Icaria/FUHEM, ver también BOLTVINIK, J. (1990). Pobreza y necesidades básicas. Conceptos $y$ métodos de medición. Caracas: PNUD.

27. Room, G. (1995). Beyond the threshold: the measurement and analysis of Social Exclusion. Bristol: The Policy Press; y los artículos DE-HaAN, A. (1998). «Social exclusion: an alternative concept for the study of deprivation». IDS Bulletin, 29, 1, enero, p. 10-19.

28. De-HaAn, A.; MaXwell, S. (1998). «Poverty and social exclusion in North and South». IDS Bulletin, 29, 1, enero, p. 1-9; TRUMAN, C. (1998). «Social exclusion and social research: towards an emancipatory framework». ISA; EVANS, M. (1998). "Behind the rhetoric: the institutional basis of social exclusion and poverty». IDS Bulletin, 29, 1, enero, p. 42-49.

29. CASTEL, R. (1995). «De la exclusión como estado a la vulnerabilidad como proceso». Archipiélago, 21, verano, p. 27-36, y especialmente el trabajo de MARTínez ROMÁn, M.A. (1997). «Política social, pobreza y exclusión social». En ALEMÁn, C.; GARCÉs, J. (coords.) (1997). Politica Social. Madrid: McGraw-Hill. 
el elemento material de la existencia, mientras que la exclusión se ha centrado en elementos más cercanos a capacitación y formación. Desde nuestro punto de vista, podemos considerarlos elementos sinónimos dentro de un mismo proceso de desarrollo (o mal desarrollo, parafraseando a José María Tortosa ${ }^{30}$ ).

Procesos de exclusión y de empobrecimiento nos hablan de los mismos elementos: aquéllos que impiden que las personas se realicen plenamente como seres humanos en todas sus dimensiones posibles y potenciales. Así, los excluidos del siglo XXI son los empobrecidos durante el siglo XX, aunque, evidentemente, las personas se concentran en hogares, grupos, comunidades, estados (unidades de análisis) y éstas nos permiten hablar de estados dentro del sistema mundial excluidos de los procesos de crecimiento o de desarrollo humano; nos permiten señalar grupos cuyas características sociológicas indican que están iniciando un proceso de empobrecimiento claro o ya están plenamente inmersos en él, etc. Procesos de empobrecimiento, desigualdad y exclusión se relacionan de una manera peculiar que se resume en que las situaciones de empobrecimiento y/o exclusión social tienen en sus bases rasgos de sociedades (locales, mundiales) desiguales. Pero situaciones de desigualdad no tienen por qué derivar en procesos de empobrecimiento.

Un ejemplo de lo anterior lo podemos constatar cuando sobre un eje cartesiano tomamos el PIB estandarizado de los diferentes estados y se cruza con la esperanza de vida (también estandarizada) de dichos países. Así, países de alto PIB presentan alta esperanza de vida y, al contrario, países de bajo PIB presentan una menor esperanza de vida. La pregunta surge cuando nos detenemos en un grupo de países en los que se da bajo PIB y niveles de esperanza de vida iguales o similares a los grupos de mayor PIB, cuando conocemos que la esperanza de vida tiene un límite superior que, de momento, no puede ser variado considerablemente.

Elementos como las políticas sociales, los modelos de acumulación y distribución de la riqueza hacen posibles estas alteraciones, y aunque son raras y con poca incidencia, sí demuestran que es posible que haya modelos de crecimiento económico e igualdad (al menos tomando como indicador la esperanza de vida).

Es interesante estudiar quiénes son los empobrecidos y sus características ${ }^{31}$, pero para pasar a la búsqueda de explicaciones plausibles de dichas situaciones de empobrecimiento (empíricas o teóricas, y que sirvan de apoyo e inicio para futuras investigaciones). Es decir, la diferencia entre estudiar los grupos de riesgo más claros que participan o participarán de situaciones de empobrecimiento, y estudiar dichos procesos y grupos desde la perspectiva de género, por ejemplo, radica en las explicaciones. Los pobres no sólo se pueden contar (muchas veces, eso carece de interés) y en el intento, comprobar que los datos nos ofrecen un porcentaje mayor de mujeres dentro de los pobres que de hom-

30. Tortosa, J.M. (1992). Sociología del Sistema Mundial. Madrid: Tecnos.

31. Ver la justificación en STREeTEN, P. «Human development-Means and ends». American Economic Review, LXXXIV, 2, p. 238-243. 
bres. Ésa es la evidencia empírica. Pero la evidencia sociológica desde la perspectiva de género ofrece interpretaciones sobre el proceso de empobrecimiento en general, con especial relación a las desigualdades de género.

Por redundar en lo evidente: diferentes enfoques, diferentes conceptos y estudios y maneras de entender la realidad social en su dimensión más dramática (empobrecimiento), ofrecen diferentes estrategias (y metodologías) para su análisis.

\section{Perspectivas de género y procesos de empobrecimiento}

La perspectiva de género no siempre ha estado presente en los análisis de la pobreza, aunque sí se venían realizando análisis parciales de los datos de que disponemos para el colectivo general, cuántos son hombres y cuántas mujeres, o niños o ancianos, etc.

En este contexto, se observa que estamos ante una visión parcial y nada explicativa del proceso de empobrecimiento. Es tan sencillo como realizar una tabla en la que se quiere saber cuántas mujeres no tienen acceso a la educación primaria, o cómo es la distribución de mujeres, dentro de aquellos hogares que tienen una renta inferior a un estándar. (Esto es particularmente complicado cuando los datos de que disponemos no hacen referencia a personas sino a hogares, por ejemplo para el caso de la EPF española u otras a nivel europeo, por lo que hay que recurrir a otro tipo de medidas de pobreza absolutas.)

Siguiendo con estas indicaciones, es posible entender conceptos como feminización de la pobreza, que hizo fortuna a finales de la década de 1980 y que sigue utilizándose en la actualidad en investigaciones de ámbito tanto internacional ${ }^{32}$ como nacional ${ }^{33}$.

También pueden verse los trabajos en esta dirección del PNUD (informes sobre desarrollo humano de 1991 y de 1995), en los que se sigue utilizando esta terminología, aunque con algunas matizaciones, en los últimos informes en los que se desarrollan algunos indicadores más cercanos a la segunda perspectiva que vamos a desarrollar a continuación.

Esta visión, que podríamos llamar mujer y pobreza, no es tan clara a la hora de tomar variables económicas en el aspecto más monetario de la pobreza. Las complicaciones metodológicas que implica, por ejemplo, calcular el número

32. Allo, F; Harcourt, W. (1997). «From South to the North; evolving perspectives on gender and poverty». Gender and development, vol. 5, 3, p. 9-12; ANDERSEN, J.; LARSEN, J. (1998). "Gender, poverty and empowerment». Critical social policy, 55, p. 241-258; Buvinic, M. (1998). "Women in poverty: a new underclass». Population and development review, 24, 1, p. 131-139 y más explícitamente MARCOUX, A. (1998). "The feminization of poverty: claims, facts and data needs». Population and devolpment review, vol. 24, 1, p. 131-139 y DAVIEST, H.; JosHI, H. (1998). "Gender and income inequality in the UK 1968-1990: the feminization of earnings or of poverty». Journal of Royal Statistical Society. Series A, vol. 161, 1, p. 33-61.

33. López larrea, M. (1989). «La feminización de la pobreza». Cuadernos de Acción Social, 14 , p. $51-55$. 
Tabla 3. Pobreza por género y estado civil del cabeza de familia (\% hogares).

\begin{tabular}{lccccc}
\hline & España, 1990 & Italia, 1995 & USA, 1994 & Francia, 1989 & Finlandia, 1995 \\
\hline Hombre soltero & 10,2 & 14,2 & 19,8 & 10,3 & 7,8 \\
Hombre casado & 8,7 & 11,6 & 9,9 & 7,5 & 1,6 \\
Mujeres solteras & 17,4 & 20,7 & 34,6 & 17,8 & 8,9 \\
Mujeres casadas & - & 37,1 & - & 28,6 & 14,8 \\
Total & 10,1 & 13,8 & 18,7 & 10,5 & 5,6 \\
\hline
\end{tabular}

Fuente: elaboración propia con datos del LIS.

de mujeres pobres con datos de la EPF en España son más que evidentes, al ser la unidad de análisis el hogar y no la mujer. Para investigaciones comparadas a nivel europeo, tenemos los datos contenidos en la base de datos del LIS (Luxembourg Income Study ${ }^{34}$ ), que reúne las equivalentes europeas a la EPF española (tabla 3).

Como ejemplo ilustrativo, cabe señalar que la simple construcción de una variable específica que sea «hogar cuyo cabeza de familia sea mujer y además tenga unos ingresos netos disponibles considerados por debajo del umbral de pobreza» es realmente complicado. Aún así se puede construir, pero tanta transformación directa de los datos originales provocan efectos perversos sobre los datos. La cuestión se complica aún más cuando se quiere ofrecer una perspectiva comparada entre estados, por ejemplo.

Aunque ésta es la visión hegemónica en las ciencias sociales, por suerte se ha venido elaborando una visión un poco antitética a la anterior desde la década de 1980 y casi de forma paralela ${ }^{35}$. Es la que habla del proceso de empobrecimiento (o bienestar, también podemos encontrar esa acepción) y género. Por decirlo de otra forma, no se trata de ver que las mujeres son pobres, sino que la pobreza está condicionada por el género ${ }^{36}$.

En primer lugar, la definición de pobreza más ligada al proceso de empobrecimiento y a términos utilizados en estas páginas es la que impera en el enfoque género y desarrollo. Si ligamos a esta visión la dificultad a la hora de obtener datos empíricos, podemos entender que este enfoque no sea precisamente el utilizado mayoritariamente. Frente a la perspectiva cuantitativa, predomina un enfoque más cualitativo. Frente al dato, a la encuesta, al agregado,

34. Ver la composición y motivos del LIS en http://www.lissy.ceps.lu/access.htm o en SMEEDING, T;; SCHMAUS, G.; AllegreZA, S. (1985). An introduction to LIS, LIS Working Papers, 1.

35. JaCKSON, C. (1996). «Rescuing Gender from the Poverty Trap». World Development, vol. 24, núm. 3, p. 489-504.

36. JACKSON, C. (1998). «Women and poverty or Gender and Well-being». Journal of International Affairs, 52, 1, p 67-81; KABEER, N. (1996). «Agency, well-being and inequality. Reflections on the gender dimensions of poverty». IDS Bulletin, vol. 27, 1; SHAFFER, P. (1998). «Gender, Poverty and Deprivation: Evidence from the Republic of Guinea». World Development, vol. 26, 12, p. 2119-2135; RAZAVI, S. (1997). «Fitting Gender into Development Institutions». World Development, vol. 25, 7, p. 1111-1125. 
prevalece un análisis más dinámico, más de proceso, de historia de vida, personalizado en el discurso de la mujer.

Para intentar obtener datos (otra vez recurrimos a funestas manías citadas con anterioridad), organismos internacionales como el PNUD ya empezaron a trabajar (en 1990), en primer lugar, en la consecución de un indicador sintético en el que se pudiera resumir gran cantidad de información, ya que se parte de la premisa que la pobreza es multidimensional y no únicamente monetaria o de rentas. Así, se creó el Índice de Desarrollo Humano (IDH), que contiene, en su configuración, elementos de renta (efectivamente, no podemos olvidar esto), esperanza de vida, niveles de escolarización, entre otros, adquiriendo un valor en una escala de 0 (mínimo desarrollo humano, luego máxima pobreza) y 1 (máximo desarrollo humano, luego mínima pobreza) ${ }^{37}$. Y aunque el ejercicio estadístico sea similar al de los enfoques de la feminización de la pobreza, el señalar cuestiones como la deforestación, agua potable disponible..., y otra serie de indicadores aún insuficientes a nuestro entender, es ya un avance.

Aunque aceptemos que el IDH es un buen indicador del empobrecimiento (que no está tan claro, pero siempre es mejor que las medidas únicamente basadas en la renta ${ }^{38}$ ), tenemos varios problemas evidentes: el primero es que es válido para un agregado internacional, tomando como unidad de análisis el estado. No sería despreciable un intento general para aplicar este tipo de indicadores sintéticos y multidimensionales para otras unidades no estatales; y, segundo, ¡cómo incluir la dimensión de género en este indicador sintético? Para ambas cuestiones tenemos algunas respuestas. Para la primera cuestión tenemos algunos ejercicios para regiones en los países de la OCDE, incluso para España ${ }^{39}$. Para la segunda cuestión no tenemos tantas respuestas.

Desde 1995 el PNUD ofrece en sus informes anuales sobre desarrollo humano, un índice que primeramente llamaron Índice de Potenciación de la Mujer y que más tarde cambiaría al actual nombre Índice de Potenciación de Género (IPG), en clara relación con lo que estamos comentando, que la mujer no es un indicador o una unidad de análisis, insertándose dentro del género, tanto hombres como mujeres.

Este indicador, cuya metodología es conocida y se puede consultar en los informes del PNUD a partir de 1995, ha sido revisado y corregido en algunos casos en sus dimensiones fundamentales, que son como en el caso del IDH, pero añadiendo la participación de la mujer en la economía y en la vida política y en la toma de decisiones.

37. Para una metodología más detallada sobre el IDH, ver los apartados metodológicos de cada uno de los informes sobre desarrollo humano que publica anualmente el PNUD. Se puede consultar el último informe (PUND, 1999). Human Development Report 1999, op. cit., p. 247-260.

38. STREETEN, P. (1998). «Beyond the six veils: conceptualizing and measuring poverty». Journal of International Affairs, 52, 1.

39. VILlOTA, P. DE (1997). «Indicadores de desarrollo humano desde una perspectiva de género». En MaQuieira, V.; VARA, Ma J. (eds.) (1997). Género, clase y etnia en los nuevos procesos de globalización. Madrid: Universidad Autónoma. 
Es curiosa la comparación de la distribución del IDH y del IPG para un mismo país y un mismo año, para comprender precisamente la dimensión de género en el proceso de empobrecimiento o en el desarrollo humano. Por ejemplo, para 1999, Canadá, que ostentaba la primera posición en la jerarquía del desarrollo humano, pasa al cuarto lugar cuando se hace referencia al Índice de Potenciación de Género. Francia (undécimo en el IDH) pasa al puesto treinta y seis en el IPG. Y, por el contrario, Suecia, que estaba en el puesto seis del IDH en 1999, pasa al segundo puesto en cuanto al desarrollo de la mujer, solamente superado por Noruega, que ostenta el primer puesto en el IPG ${ }^{40}$.

Dicho de otra manera, parece que desde un punto de vista metodológico, el IPG introduce elementos cercanos a la dimensión de género y desarrollo, aunque no es del todo satisfactorio conceptualmente y desde la perspectiva de género, principalmente por la fuente y la naturaleza únicamente cuantitativa de los datos. Corregir, o mejor, ampliar la información de los sistemas de indicadores mundiales e incorporar elementos de carácter más local vinculados no sólo al estado, sino a otros tipos de agregados, es un trabajo el cual deberíamos estar desarrollando ya hace un tiempo. Pero no es así. Como tampoco es evidente la inclusión de la perspectiva cualitativa (aunque muchas veces se haya pedido) en los estudios sobre el empobrecimiento y el género, desde un quehacer metodológico y científico riguroso y lejos de las aventuras literarias que han proliferado en los últimos tiempos.

Si el estudio de la pobreza desde la perspectiva de género es, muchas veces y por la bibliografía disponible, un intento de movilización de la mujer en los países pobres a través de la activación de recursos no convencionales, es comprensible que tengamos cortapisas a la hora de diseñar investigaciones empíricas metodológicamente hablando. Tampoco sabemos si es ello un problema. Más bien es un argumento argüido por quienes no entienden que la pobreza es femenina y los pobres, masculinos, semánticamente hablando.

\section{Conclusiones y alternativas para una presencia nada clara}

Los desenfoques a la hora de estudiar la pobreza se reflejan en la confusión conceptual, en la utilización de un solo paradigma de análisis, en el reduccionismo frente a la complejidad de las situaciones sociales. Básicamente los (des)enfoques con los que hemos venido analizando los procesos de empobrecimiento masivos han sido definidos y aplicados desde un punto de vista masculino. Y masculinos son los indicadores y masculinos los resultados de las investigaciones. Y, por lo tanto, son masculinas las propuestas, cuando las hay, en la lucha contra la pobreza, siendo el ejemplo claro de lo anterior para España, las políticas familiares para fortalecer un supuesto colchón amortiguador de la pobreza, que no es tal, ya que la familia se basa en una estructura patriarcal y en una desigual redistribución de los recursos en su seno. 
La ausencia de un discurso femenino en los estudios sobre procesos de empobrecimiento es patente. No nos referimos a las voces de investigadoras que trabajen en la medición de la pobreza desde una perspectiva de ingresos o gastos del hogar familiar. Nos referimos a la ausencia de la perspectiva de género a la hora de la definición de las situaciones de pobreza, de los procesos de empobrecimiento. En definitiva, nos referimos a la voz de aquéllas que, pobres o no, empobrecidas o con un medio pasar, han sido sistemáticamente excluidas de los diseños metodológicos.

Abogamos por la inclusión real de los aspectos relacionados con el género y que no han estado presentes en la investigación social, empezando por una definición consensuada y más rica del proceso de empobrecimiento que tenga como nota, ya no discordante, sino unificadora, la voz de las mujeres. Ello requiere metodologías y diseños integrados, unidades y variables diferentes a las actuales (no sólo las actuales) y propuestas técnicas (indicadores compuestos, fundamentalmente) que no sean únicamente extensión estadística y reformulación paramétrica de unos datos pensados para analizar realidades sociales en las que la perspectiva de género está ausente.

Y todo ello con un claro objetivo aplicado: reducir los procesos de empobrecimiento que parecen haberse disparado en la última década. Porque sabemos (tenemos evidencia empírica) que son las iniciativas de las mujeres las que reducen la pobreza y las que mitigan los procesos de empobrecimiento. 